\title{
99mTc SPECT/CT Versus Planar Lymphoscintigraphy for Preoperative Sentinel Lymph Node Detection in Cervical Cancer: A Systematic Review and Metaanalysis
}

\author{
Jacob P. Hoogendam ${ }^{1}$, Wouter B. Veldhuis ${ }^{2}$, Monique G.G. Hobbelink ${ }^{2}$, René H.M. Verheijen ${ }^{1}$, Maurice A.A.J. van den \\ Bosch $^{2}$, and Ronald P. Zweemer ${ }^{1}$ \\ ${ }^{1}$ Department of Gynaecological Oncology, University Medical Center Utrecht, Utrecht, The Netherlands; and ${ }^{2}$ Department of \\ Radiology and Nuclear Medicine, University Medical Center Utrecht, Utrecht, The Netherlands
}

\begin{abstract}
We aimed to compare SPECT/CT and lymphoscintigraphy on overall and bilateral sentinel lymph node (SLN) detection in cervical cancer patients. Methods: A systematic search was performed on August 1, 2014, in PubMed, Embase, Scopus, and the Cochrane library. The syntax was based on synonyms of the terms cervical cancer, SPECT/CT, and lymphoscintigraphy. Retrieved articles were screened on their title/abstract and considered eligible when an SLN procedure was performed using both imaging modalities and if detection results were reported. Two independent reviewers assessed all included studies on methodologic quality using QUADAS-2. Studies were pooled on their odds ratios (ORs) with a randomeffects model. Results: The search yielded 962 unique articles, of which 8 were ultimately included. The studies were recent retrospective or prospective cohort studies of limited size $(n=7-51)$ but sufficient methodologic quality. The median overall detection ( $\geq 1$ SLN in a patient) was $98.6 \%$ for SPECT/CT (range, $92.2 \%-$ $100.0 \%$ ) and $85.3 \%$ for lymphoscintigraphy (range, $70.0 \%-$ $100.0 \%)$. This corresponded to a pooled overall SLN detection OR of $2.5(95 \% \mathrm{Cl}, 1.2-5.3)$ in favor of SPECT/CT. The reported median bilateral detection ( $\geq 1 \mathrm{SLN}$ in each hemipelvis) was $69.0 \%$ for SPECT/CT (range, $62.7 \%-79.3 \%$ ) and $66.7 \%$ for lymphoscintigraphy (range, 56.9\%-75.8\%), yielding a pooled OR of 1.2 $(95 \% \mathrm{Cl}, 0.7-2.1)$. No significant difference in the number of visualized SLNs was observed at a pooled ratio of $1.2(95 \% \mathrm{Cl}, 0.9-1.6)$. Conclusion: In cervical cancer patients, preoperative SLN imaging with SPECT/CT results in superior overall SLN detection in comparison with planar lymphoscintigraphy.
\end{abstract}

Key Words: SPECT-CT; lymphoscintigraphy; sentinel lymph node; cervical cancer; meta-analysis

J Nucl Med 2015; 56:675-680

DOI: 10.2967/jnumed.114.152439

I recent years, the sentinel lymph node (SLN) procedure has been increasingly adopted in the staging of cervical cancer patients eligible for surgery. The SLN procedure allows for individualized treatment decisions by accurately ascertaining the lymph

Received Dec. 2, 2014; revision accepted Mar. 16, 2015.

For correspondence contact: Jacob P. Hoogendam, UMC Utrecht, F05. 126, P.O. Box 85500, 3508GA, Utrecht, The Netherlands.

E-mail: j.hoogendam@umcutrecht.nl

Published online Apr. 9, 2015.

COPYRIGHT (C 2015 by the Society of Nuclear Medicine and Molecular Imaging, Inc. node status before radical surgery takes place (1), with the possibility of excluding fertility-sparing surgery or replacing a radical hysterectomy with chemoradiotherapy in patients with tumorpositive lymph nodes.

Besides blue dye, the colloid-bound radionuclide ${ }^{99 \mathrm{~m} T c}$ is commonly added as a second tracer and has been shown to improve intraoperative SLN detection (2). A second advantage of this tracer is that preoperative lymphatic mapping by either SPECT/ CT or planar lymphoscintigraphy becomes possible, enabling prediction of the surgical detectability and number of SLNs in an individual patient. This ability aids the surgeon in performing a more direct SLN resection with less disruption of the lymphatic architecture when compared with a full retroperitoneal exploration (3-5).

Although SPECT/CT is associated with both increased upfront cost and ionizing radiation, it provides a cross-sectional anatomic reference that allows for accurate 3-dimensional SLN localization, which is considered an important advantage over planar lymphoscintigraphy (5-9). Both SPECT/CT and lymphoscintigraphy should ideally have a high SLN detection ability, which largely determines the clinical value of the entire procedure.

On the basis of a systematic search of the literature, we compared detection of SLNs on preoperative mapping by SPECT/CT and lymphoscintigraphy in cervical cancer patients. Through a metaanalysis of the retrieved studies, we aimed to quantify both the overall and the bilateral SLN detection differences between these 2 imaging modalities.

\section{MATERIALS AND METHODS}

\section{Systematic Search}

We conducted a systematic review and metaanalysis of the medical literature in adherence with the PRISMA guideline (Preferred Reporting Items for Systematic Reviews and Meta-Analyses) (10). Before the search was initiated, a protocol was devised that specified the research question, search strategy, inclusion and exclusion criteria, quality assessment, data collection, and statistical analysis.

A title- and abstract-based literature search was simultaneously performed on August 1, 2014, for 4 online databases: PubMed/ MEDLINE, Embase, Scopus, and the Cochrane Library. To attain a comprehensive search, the syntax was based on multiple synonyms, abbreviations, and common adjectives for the search terms representing our study domain (i.e., population) and determinants (i.e., intervention and comparison). These search terms were cervical cancer, SPECT/CT, and lymphoscintigraphy. The outcome measure was deliberately omitted from the search, given that this could be 
(Cervical cancer [Title/Abstract] OR Cervical tumor [Title/Abstract] OR Cervical carcinoma [Title/Abstract] OR Cervical malignancy [Title/Abstract] OR Cervical neoplasm [Title/Abstract] OR Cervical [Title/Abstract] OR Cancer of the uterine

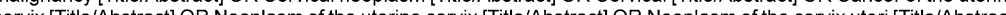
OR [ [Tin

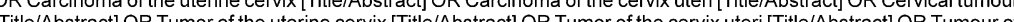

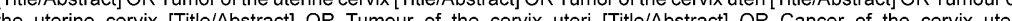
[The uterine cervix [Tile/Abstract] OR Tumour of the cervix uni [Title/Abstract] OR Cancer of the cervix uteri [Title/Abstract] OR Malignancy of the uterine cervix [Title/Abstract] OR Malignancy of the cervix uteri [Title/Abstract]

AND

Single photon emission computed tomography [Title/Abstract] OR Single photon emission computed tomographic [Title/Abstract] OR Single photon emission computerized tomography [Title/Abstract] OR Single photon emission [rite/Abstict computerised tomography [Title/Abstract] OR Single photon computed tomography [Title/Abstract] OR Single photon computed tomographic [Title/Abstract] OR SPECT [Title/Abstract] OR SPECT-CT [Title/Abstract] OR SPECT/CT [Title/Abstract] OR SPETICT [Title/Abstract] OR Hybrid Title/Abstract] OR Computed tomography [Title/Abstract] OR CT [Title/Abstract] OR CAT [Title/Abstract])

AND

(Lymphoscintigraphy [Title/Abstract] OR Lymphoscintigraphies [Title/Abstract] OR Lymphoscintigraphs [Title/Abstract] OR Lymphoscintigram [Title/Abstract] OR Lymphoscintigrams [Title/Abstract] OR Scintigraphy [Title/Abstract] OR Scintigraphies [Title/Abstract] OR Scintigraphs [Title/Abstract] OR Scintigram [Title/Abstract] OR Scintigrams [Title/Abstract] OR LSG [Title/Abstract] OR Planar [Title/Abstract] OR Conventional imaging [Title/Abstract] OR Gamma [Title/Abstract] OR Lymphatic mapping [Title/Abstract] OR Lymphatic imaging [Title/Abstract] OR Nuclear medicine imaging [Title/Abstract]]

FIGURE 1. PubMed/MEDLINE search syntax.

a secondary finding in some articles and not included in the title or abstract. Figure 1 outlines the exact search syntax used. No medical subject headings, filters, or publication date limits were used.

All identified references were exported to the online reference management software RefWorks-COS (ProQuest LLC) for removal of duplicate articles.

\section{Eligibility Assessment}

The title and abstract of all unique articles were screened by a single reviewer to determine eligibility. The inclusion of individual studies required that an SLN procedure had been performed on cervical cancer patients with preoperative SLN mapping by both SPECT/CT and lymphoscintigraphy within the same study. Also, the study had to have reported unilateral or bilateral detection results for both these imaging modalities. To allow a valid comparison, we aimed to include only studies that directly compared both techniques. Studies were excluded from the review when they did not contain original data or were not written in English, Dutch, German, or French. Conference

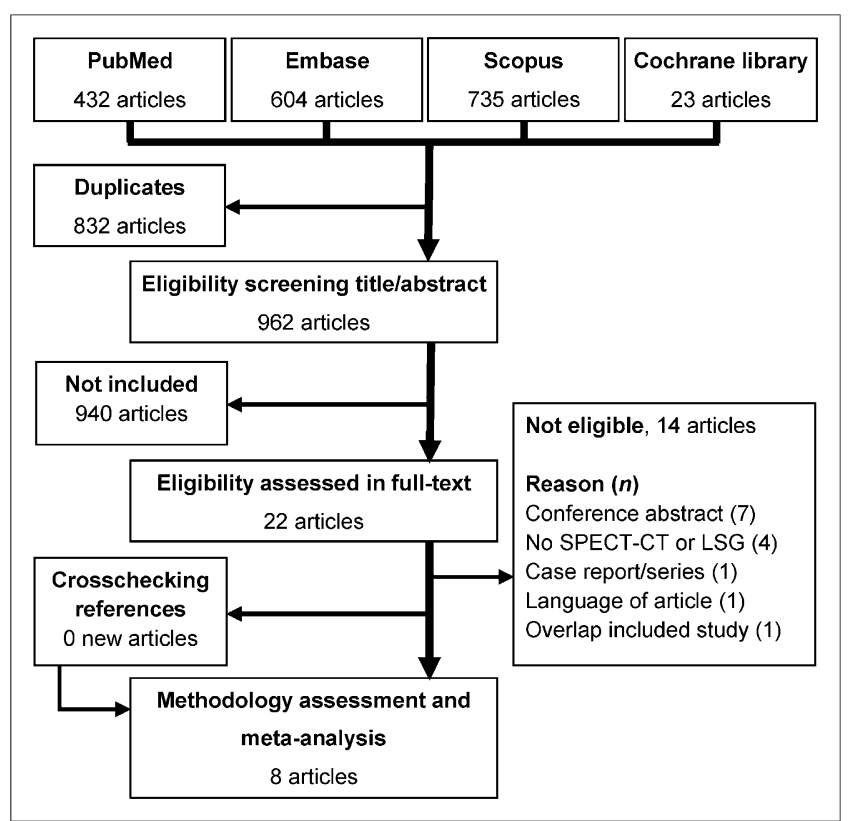

FIGURE 2. Flow diagram of search and study assessment, with number of articles at each stage. abstracts and case reports or series with no more than 3 valid cases were also excluded. When multiple eligible articles reported on the same patients, only the article demonstrating the most comprehensive results with respect to our research question was selected.

Full-text assessment was performed when the eligibility of an article, based on title and abstract screening, remained uncertain. Articles deemed eligible on the basis of initial screening were also read in full-text, during which their eligibility was rechecked. The references of all included studies were carefully cross-checked with our initial search result for possible additional literature.

\section{Quality Assessment and Data \\ Collection}

A structured quality assessment of all included studies was performed using the Quality Assessment of Diagnostic Accuracy Studies instrument, version 2 (QUADAS-2) (11). QUADAS-2 is designed for grading individual diagnostic studies on their respective risk of bias and applicability in the context of systematic reviews and metaanalyses. In adherence with the QUADAS-2 recommendations, signaling questions were optimized for our research aim, though without changing overall content or structure. The critical appraisal was independently performed by 2 reviewers. Discrepancies between them were resolved via consensus discussion or, when unresolvable, by an independent third referee.

The data from the original studies were collected by a single reviewer using a standardized form, which was created in advance. This form contained variables on the research question, study design, study population, imaging modalities, intraoperative SLN procedure, and outcome level effects.

\section{Statistical Analysis}

All analyses were performed using the statistical software $\mathrm{R}$, version 3.0.3 (R Foundation for Statistical Computing); we installed the package "meta," version 3.7-1, and used it for all metaanalyses and corresponding plots.

The main outcome measures were the differences between SPECT/CT and planar lymphoscintigraphy for overall ( $\geq 1$ SLN in a patient) and bilateral ( $\geq 1$ SLN in each hemipelvis) SLN detection. For both outcomes, an inverse variance-weighted random effects metaanalysis was performed to pool the original studies on the basis of their odds ratios (ORs) (12). Studies with zero values in any of the boxes in the 2 by 2 tables underlying OR calculation were continuity-corrected $(n+0.5)$. To facilitate easy interpretation of the ORs and their clinical relevance, pooled ORs and median lymphoscintigraphy detection ratios were used to transform the pooled ORs into a percentage.

As a secondary analysis, the difference in numbers of SLNs detected between the 2 imaging modalities was analyzed. These constitute count-type data for which an inverse variance-weighted random effects metaanalysis was performed on the basis of incidence (i.e., detection) rate ratios (12). Forest plots were created to summarize all studies, the pooled estimate, and corresponding 95\% confidence intervals (95\% CIs) in a single overview.

The heterogeneity of the results from the original studies was tested by calculating the Cochrane Q-value, which follows a $\chi^{2}$ distribution. Its derived percentage $\mathrm{I}^{2}$ was calculated and used to represent the variability of results relative to chance. The between-study variance is presented by the $\tau^{2}$ statistic. Statistical significance was defined as a $P$ value of less than 0.05 . 


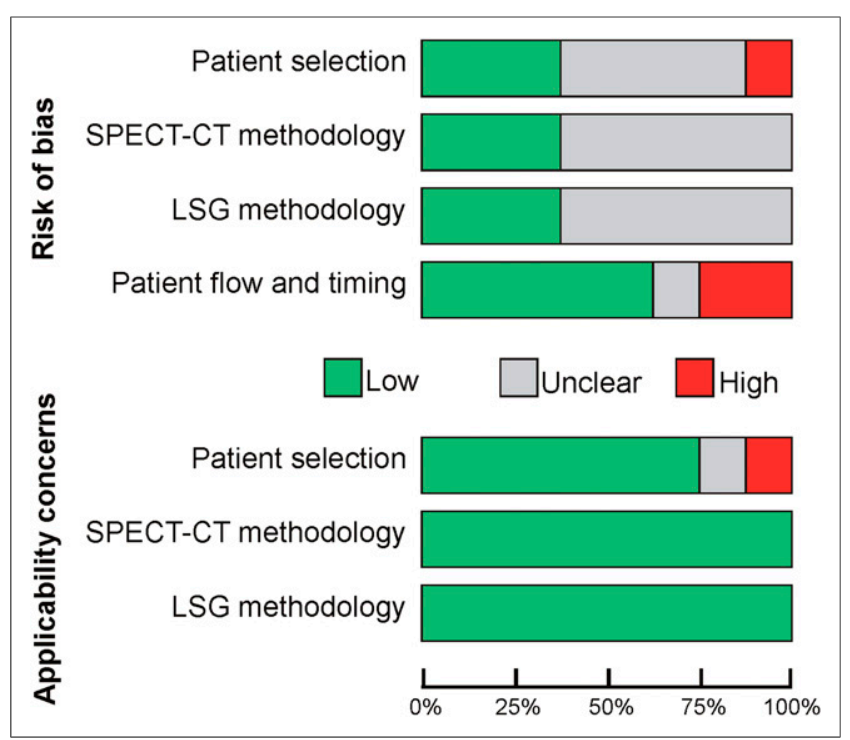

FIGURE 3. Summary of methodologic quality scored according to QUADAS-2.

\section{RESULTS}

\section{Literature Search and Assessment}

The 4 databases yielded 432 (PubMed), 604 (Embase), 735 (Scopus), and 23 (Cochrane Library) articles (Fig. 2). After the removal of duplicates, 962 unique articles were screened on their eligibility. Full-text assessment was needed for 22 articles, of which 4 did not meet the inclusion criteria because either SPECT/CT or lymphoscintigraphy was not used (13-15) or the SLN detection results were not reported for both modalities (6). A further 9 studies were excluded because of their language (16), because they were a conference abstract (17-23), or because they were a case report or series (24). One article (25) was discarded because of an overlap in patients with an included study (8). The references cited in the remaining 8 articles were cross-checked and did not yield any additional eligible studies $(3-5,8,9,26-28)$.

These 8 articles were QUADAS-2-assessed (Fig. 3). The risk of bias mainly was scored as low or remained unclear because of absence of reporting on patient inclusion criteria, consecutiveness of inclusions, or details of the SPECT/CT and lymphoscintigraphy procedures. None of the 7 paired studies specified whether and how blinding between the 2 imaging modalities was ensured. No real concerns on the applicability of studies for this metaanalysis existed.

\section{SLN Detection}

The characteristics of the studies included in the metaanalysis are outlined in Table 1. Three studies (3-5) exclusively investigated patients with cervical cancer; others did so in combination with endometrial cancer $(9,26,27)$, vulvar cancer $(28)$, or both $(8)$. In total, SPECT/CT and lymphoscintigraphy were performed for 207 and 208 cervical cancer patients, respectively. Except for a single International Federation of Gynecology and Obstetrics (FIGO) Stage IIIA case from Klapdor et al. (5), all these subjects had early-stage disease (FIGO stage I/II). Lymph node involvement for cervical cancer was reported in all studies except that of Kraft and Havel (8) and ranged from $0.0 \%$ to $28.6 \%$ (overall mean, $19.8 \%)(3-5,9,26-28)$. The outlier of $0 \%$ lymph node metastasis occurred in a single study with 10 patients (26).
All included studies with the exception of Díaz-Feijoo et al. (3) reported a higher overall SLN detection for SPECT/CT than for lymphoscintigraphy. The median overall detection was $98.6 \%$ for SPECT/CT (range, $92.2 \%-100.0 \%$ ) and $85.3 \%$ for lymphoscintigraphy (range, $70.0 \%-100.0 \%$ ). At the pooled level, a statistically significant OR of 2.5 (95\% CI, 1.2-5.3) was detected, favoring SPECT/CT (Fig. 4). The consistency of the detection results was confirmed by the negligible heterogeneity across the included studies. On the basis of the median $85.3 \%$ overall SLN detection for lymphoscintigraphy and the pooled OR, a calculated $93.6 \%$ (95\% CI, 87.3\%-96.9\%) overall detection on SPECT/CT should be achieved. This equals a detection increase of $8.3 \%$ (95\% CI, $2.0 \%-11.6 \%)$.

Three of the 8 studies reported bilateral SLN detection ratios, covering a total of 122 (SPECT/CT) and 123 (lymphoscintigraphy) patients. The median bilateral detection was $69.0 \%$ for SPECT/CT (range, $62.7 \%-79.3 \%$ ) and $66.7 \%$ for lymphoscintigraphy (range, $56.9 \%-75.8 \%)(4,5,9)$. A pooled OR of $1.2(95 \% \mathrm{CI}$, 0.7-2.1) was detected for bilateral SLN detection, indicating absence of a significant difference (Fig. 5). This equals a $4.1 \%$ bilateral detection difference $(95 \% \mathrm{CI},-8.1 \%$ to $14.0 \%)$.

Five studies could be pooled on the number of SLNs detected by both modalities $(3,4,9,26,28)$. On SPECT/CT, 345 SLNs were visualized in 110 patients, relative to 299 SLNs in 111 patients on lymphoscintigraphy. The pooled ratio in SLN count was $1.2(95 \%$ CI, 0.9-1.6), which reflects no significant increase in the number of SLNs detected on SPECT/CT (Fig. 6).

\section{DISCUSSION}

All 8 original studies showed excellent and consistent overall SLN detection ratios for SPECT/CT, ranging from $92.0 \%$ to $100.0 \%$. Its pooled SLN detection was superior to that of lymphoscintigraphy, with an OR of 2.5 (95\% CI, 1.2-5.3), corresponding to a relevant $8.3 \%$ increase $(95 \% \mathrm{CI}, 2.0 \%-11.6 \%)$. Clinically, this means that of the median $14.7 \%$ nonvisualization cases on lymphoscintigraphy, more than half can be prevented through the use of SPECT/CT (6.4\% nonvisualization). Its significantly improved overall SLN detection supplements other potential advantages. The cross-sectional nature of SPECT/CT and the anatomic reference it provides has been reported-though rarely formally analyzed - to lead to better anatomic SLN localization (4,6-8). The lack of this ability has always been regarded as a weakness of lymphoscintigraphy (29). In addition, a single report has indicated a possible reduction of $25.4 \mathrm{~min}$ for intraoperative SLN retrieval time by robot-assisted laparoscopy when preoperative SPECT/CT is used (4).

The cervix is a midline organ with bilateral lymphatic drainage, making identification of minimally 1 SLN in each hemipelvis clinically relevant (1,6,30-32). Unfortunately, only 3 studies specifically reported bilateral SLN detection results, leading to a metaanalysis of insufficient statistical power to prove or disprove a difference. The wide $95 \%$ CI $(0.7-2.1)$ relative to the limited OR of 1.2 indicates that further research is needed before any conclusive statement can be made. In general, researchers should be urged to always report bilateral SLN results in cervical cancer, even though these are often substantially lower and less attractive than the overall detection ability.

Some investigators use ${ }^{99 \mathrm{~m}} \mathrm{Tc}$-nanocolloid and blue dye via a cervical injection for the sentinel procedure in endometrial cancer patients. Although the validity and reliability are not without 


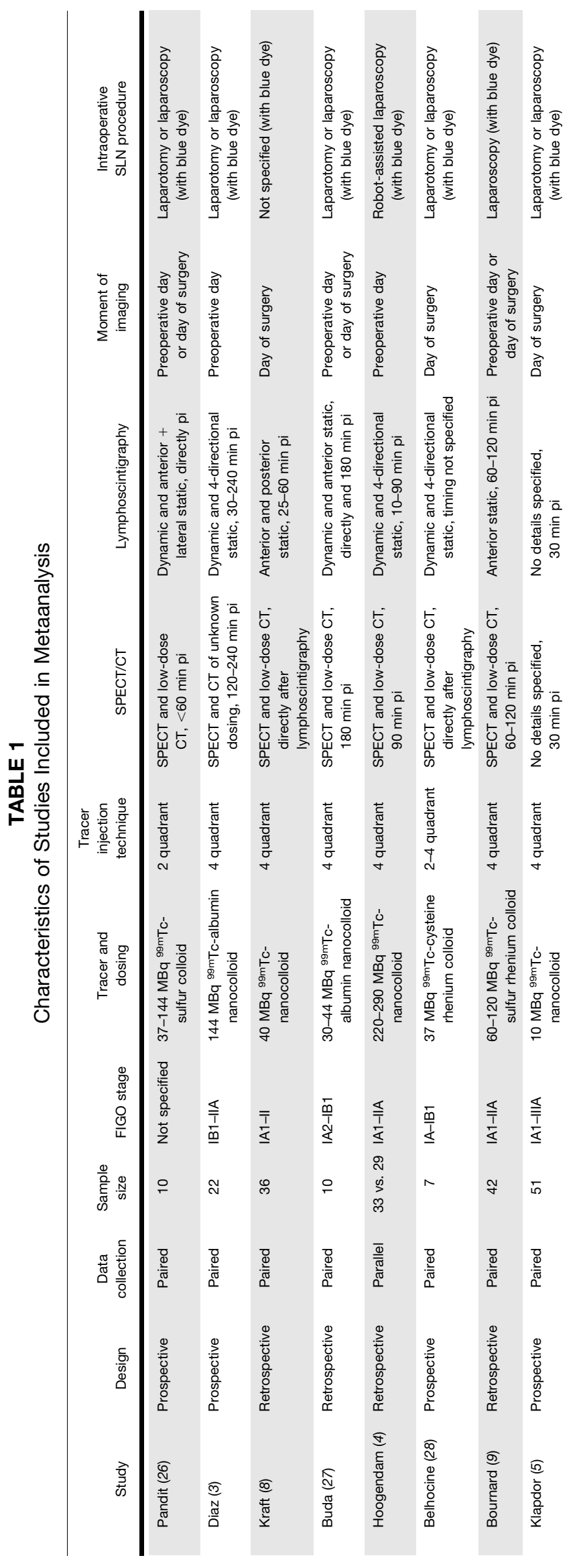

debate for this indication, the methodology is similar to the cervical cancer approach and identifies the SLNs of the cervix uteri. Four such studies $(n=21-40)$ from our initial search reported the overall SLN detection results of both SPECT/CT and lymphoscintigraphy, which ranged between $84.6 \%-100.0 \%$ and $40.0 \%-$ $85.9 \%$, respectively $(8,9,26,27)$. In addition, Cordero García et al. demonstrated an overall detection at the first imaging session of $77.8 \%(n=14 / 18)$ on SPECT/CT, compared with $73.7 \%(n=14 / 19)$ for planar lymphoscintigraphy in stage IA-IIIA endometrial cancer patients (33). Although these results, especially for lymphoscintigraphy, are lower than the cervical cancer estimates used in our metaanalysis, the superiority of SPECT/CT is maintained.

By definition, the SLN is the first efferent nodal station to receive lymphatic drainage from the tumor and therefore represents the overall lymph node status. Consequently, some authors question the need for routine pelvic lymphadenectomy when tumornegative SLNs are identified in cervical cancer $(30,32,34,35)$. Although the yet-unpublished randomized SENTICOL-2 trial (Ganglion Sentinelle dans le Cancer du Col) primarily studies complications after an SLN procedure, as opposed to after full lymphadenectomy, oncologic safety is a secondary endpoint of that study (36). When the 2 procedures are found to be equal, abstaining from systematic lymphadenectomy would safeguard lymph node-negative patients from an associated $2 \%$ intraoperative risk of vascular, nerve, bowel, or ureteric injury (37). Also, long-term morbidity caused by lymphedema and infected lymphocysts can be minimized and will likely improve quality of life $(30,38)$

The ability to safely abstain from systematic lymphadenectomy also depends on the reliability of the SLN procedure. Besides the high SLN detection shown in this metaanalysis, the reliability of the SLN procedure is determined mainly by the intraoperative risk of a false-negative diagnostic outcome (i.e., misclassifying a patient with lymph node metastases). A large multicenter study $(n=645)$ by Cibula et al. found a false-negative SLN ratio of $1.3 \%$ when bilateral detection with histopathologic ultrastaging was performed (1).

Several arguments offer a rationale for the improved SLN detection by SPECT/CT. In addition to its higher spatial resolution, it provides an anatomic reference and has a cross-sectional nature, reducing the number of overlooked SLNs near the injection depot (e.g., parametrial SLNs) and at unusual anatomic locations (e.g., paravesical, epigastric, or presacral SLNs) $(5,6,28)$. Furthermore, SPECT undergoes superior attenuation correction through the availability of concurrent CT data (39). This CT-based correction reduces the inherent overestimation of peripheral background activity (i.e., noise), leading to more valid ${ }^{99 \mathrm{~m}} \mathrm{Tc}$ tracer uptake quantification in the SPECT dataset (40).

Some limitations at the level of the original studies merit further explanation. First, in studies with a 


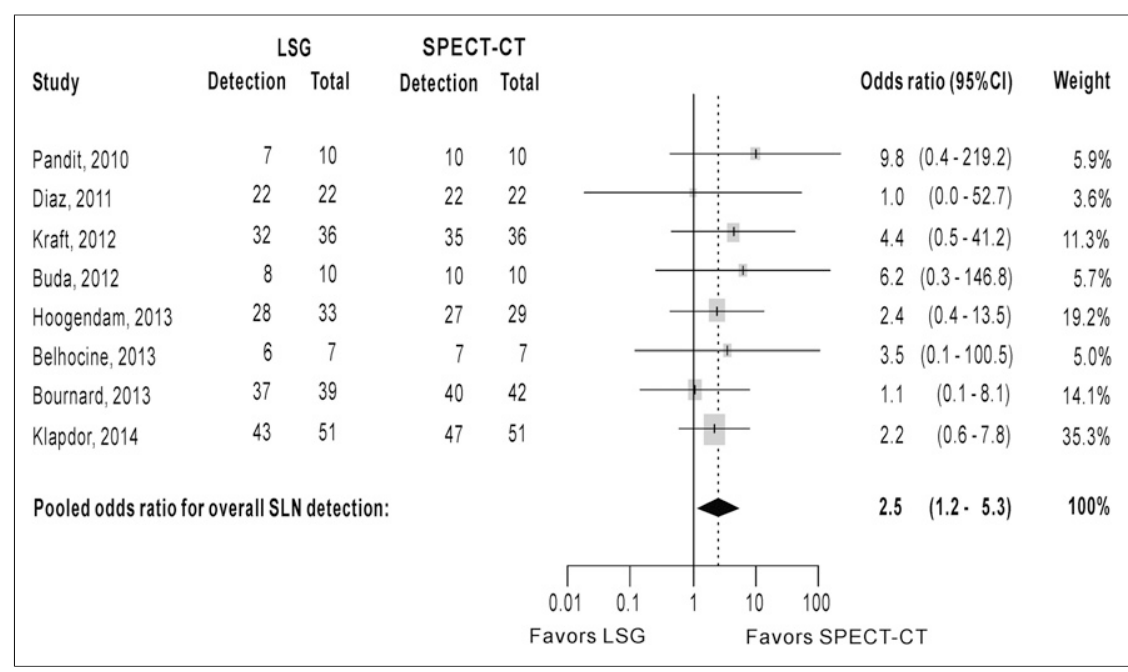

FIGURE 4. Forest plot of overall SLN detection ( $\geq 1 \mathrm{SLN}$ detected in a patient) on SPECT/CT compared with lymphoscintigraphy. Heterogeneity statistics: Cochrane $Q=2.3$ (7 degrees of freedom; $P=0.945), I^{2}=0.0 \%$, and $t^{2}=0.0$. Pandit et al. reported conflicting detection ratios of $70.0 \%$ vs. $80.0 \%$ for overall SLN detection on lymphoscintigraphy. Most conservative estimate was selected (26).

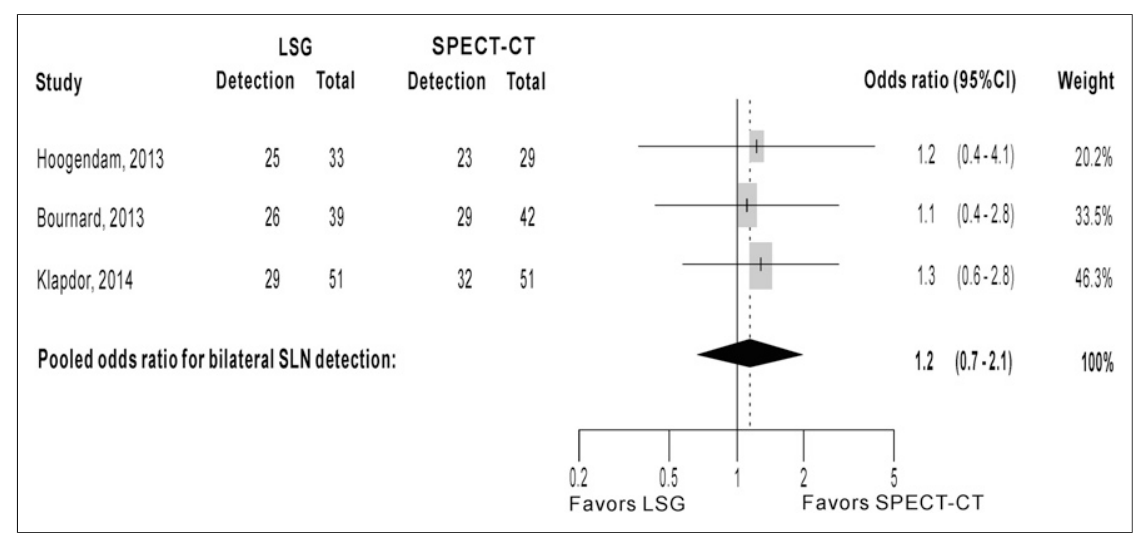

FIGURE 5. Forest plot of bilateral SLN detection ( $\geq 1$ SLN detected in each hemipelvis) on SPECT/CT compared with lymphoscintigraphy. Heterogeneity statistics: Cochrane $Q=0.1$ (2 degrees of freedom; $P=0.976$ ), $\mathrm{I}^{2}=0.0 \%$, and $\tau^{2}=0.0$. Belhocine et al. reported bilateral SLN detection in $71.4 \%(n=5 / 7)$ without specifying imaging modality by which this was achieved and was therefore excluded from this subanalysis (28).

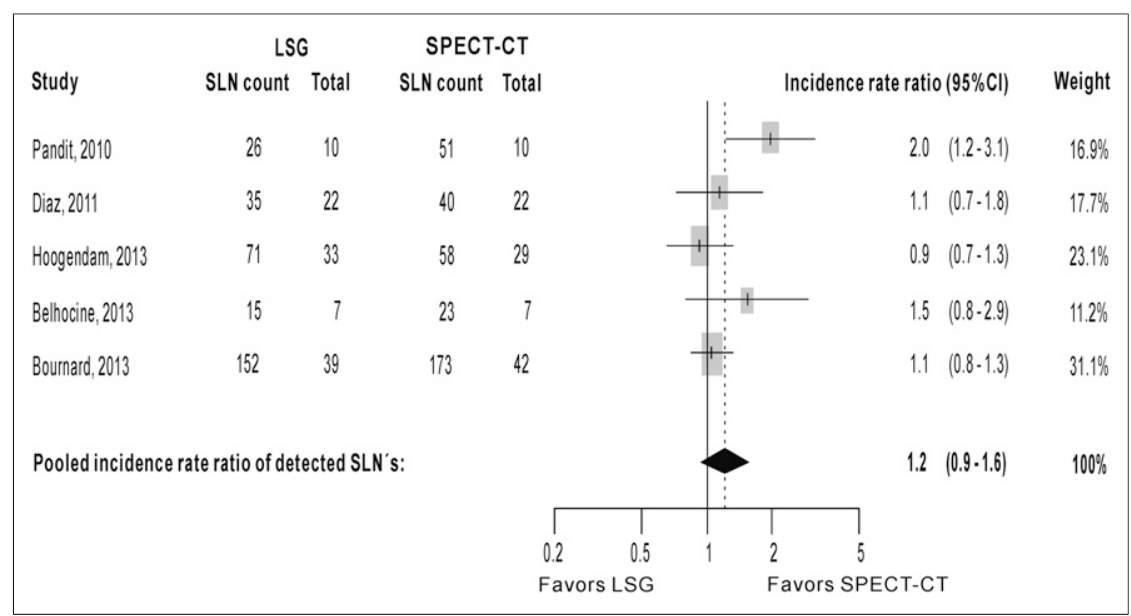

FIGURE 6. Forest plot of number of SLNs detected on SPECT/CT compared with planar lymphoscintigraphy. Heterogeneity statistics: Cochrane $Q=7.7$ (4 degrees of freedom; $P=0.105$ ), $\mathrm{I}^{2}=47.8 \%$, and $\tau^{2}=0.0$. paired design, adequate blinding during the review of the 2 imaging modalities is of the utmost importance for an unbiased comparison. However, none of the 7 paired studies had a blinded design. Lack of masking could possibly favor SPECT/CT because it is commonly performed after lymphoscintigraphy. Second, all available studies had an observational design (4 prospective and 4 retrospective) with a relatively small sample size. The limited number of original studies did not permit a formal sensitivity analysis in which only studies with a low risk of bias are pooled and compared with the effect derived from all studies. Instead, we aimed to clearly assess the methodologic quality and pool all studies.

A limitation of this study is our choice of the outcome measure. Although detection on preoperative imaging is relevant and insightful, a more clinically important outcome measure would be differences in intraoperative SLN resection. However, such an outcome measure is currently not possible since all but one original study in our metaanalysis followed a paired design and consequently had identical intraoperative SLN resection results for both imaging modalities. The single parallel study included did not report a statistically significant difference between SPECT/CT and lymphoscintigraphy for the overall $(93.1 \%$ vs. $93.9 \%)$ or bilateral $(89.7 \%$ vs. $84.8 \%)$ intraoperative SLN resection (4).

A limitation of our metaanalysis on the number of SLNs detected, which follows a Poisson distribution, is the absence of a uniformly accepted summary statistic for count-type data. We followed a common approach in which pooling was based on the rate ratio (i.e., detection rate of lymphoscintigraphy divided by detection rate of SPECT/CT), instead of treating count-type data as a continuous variable (12). However, in detection rate calculation the SLN count should be divided by person-time, a nonexistent entity in our imaging setting, which was therefore replaced by the respective number of scans made.

\section{CONCLUSION}

In cervical cancer patients, higher overall SLN detection is provided by preoperative SPECT/CT than by lymphoscintigraphy. Larger studies are still needed before any significant conclusions on bilateral detection, or the number of SLNs visualized, can be reached. Nonetheless, the overall detection ability is high and the difference from lymphoscintigraphy substantial. The reduction in 
cases of nonvisualized SLNs by more than half through the use of SPECT/CT is a relevant clinical improvement. This advantage supplements the other advantages of SPECT/CT, including possibly shortened surgical SLN retrieval times and more precise preoperative information on the anatomic location of the SLNs. In view of these combined results, few reasons remain for continuing the use of planar lymphoscintigraphy for SLN detection in cervical cancer when SPECT/CT is available.

\section{DISCLOSURE}

The costs of publication of this article were defrayed in part by the payment of page charges. Therefore, and solely to indicate this fact, this article is hereby marked "advertisement" in accordance with 18 USC section 1734 . No potential conflict of interest relevant to this article was reported.

\section{ACKNOWLEDGMENT}

We thank Rob J.P. Scholten as head of the Dutch Cochrane Center for his advice on the manuscript.

\section{REFERENCES}

1. Cibula D, Abu-Rustum NR, Dusek L, et al. Bilateral ultrastaging of sentinel lymph node in cervical cancer: lowering the false-negative rate and improving the detection of micrometastasis. Gynecol Oncol. 2012;127:462-466.

2. van de Lande J, Torrenga B, Raijmakers PG, et al. Sentinel lymph node detection in early stage uterine cervix carcinoma: a systematic review. Gynecol Oncol. 2007;106:604-613.

3. Díaz-Feijoo B, Pérez-Benavente MA, Cabrera-Diaz S, et al. Change in clinical management of sentinel lymph node location in early stage cervical cancer: the role of SPECT/CT. Gynecol Oncol. 2011;120:353-357.

4. Hoogendam JP, Hobbelink MG, Veldhuis WB, Verheijen RH, van Diest PJ, Zweemer RP. Preoperative sentinel node mapping with ${ }^{99 \mathrm{~m}} \mathrm{Tc}-$ nanocolloid SPECT-CT significantly reduces the intraoperative sentinel node retrieval time in robot assisted laparoscopic cervical cancer surgery. Gynecol Oncol. 2013;129:389-394.

5. Klapdor R, Mücke J, Schneider M, et al. Value and advantages of preoperative sentinel lymph node imaging with SPECT/CT in cervical cancer. Int J Gynecol Cancer. 2014;24:295-302.

6. Martínez A, Zerdoud S, Mery E, Bouissou E, Ferron G, Querleu D. Hybrid imaging by SPECT/CT for sentinel lymph node detection in patients with cancer of the uterine cervix. Gynecol Oncol. 2010;119:431-435.

7. Holman LL, Levenback CF, Frumovitz M. Sentinel lymph node evaluation in women with cervical cancer. J Minim Invasive Gynecol. 2014;21:540-545.

8. Kraft O, Havel M. Detection of sentinel lymph nodes in gynecologic tumours by planar scintigraphy and SPECT/CT. Mol Imaging Radionucl Ther. 2012;21:47-55.

9. Bournaud C, Le Bail-Carval K, Scheiber C, de Charry C, Mathevet P, MoreauTriby C. Value of SPECT/CT in lymphatic mapping in cervix and endometrial cancer. Médecine Nucléaire. 2013;37:387-396.

10. Moher D, Liberati A, Tetzlaff J, Altman DG. Preferred reporting items for systematic reviews and meta-analyses: the PRISMA statement. Ann Intern Med. 2009;151:264-269.

11. Whiting PF, Rutjes AW, Westwood ME, et al. QUADAS-2: a revised tool for the quality assessment of diagnostic accuracy studies. Ann Intern Med. 2011;155:529-536.

12. Higgins JPT, Green S, eds. Cochrane handbook for systematic reviews of interventions: version 5.1.0. Cochrane website. www.cochrane-handbook.org. Updated March 2011. Accessed March 19, 2015.

13. Kushner DM, Connor JP, Wilson MA, et al. Laparoscopic sentinel lymph node mapping for cervix cancer: a detailed evaluation and time analysis. Gynecol Oncol. 2007;106:507-512.

14. Feigen M, Crocker EF, Read J, Crandon AJ. The value of lymphoscintigraphy, lymphangiography and computer tomography scanning in the preoperative assessment of lymph nodes involved by pelvic malignant conditions. Surg Gynecol Obstet. 1987;165:107-110.

15. Hauspy J, Verkinderen L, De Pooter C, Dirix LY, van Dam PA. Sentinel node metastasis in the groin detected by technetium-labeled nannocolloid in a patient with cervical cancer. Gynecol Oncol. 2002;86:358-360.
16. Zhang WJ, Zheng R, Wu LY, Li XG, Li B, Chen SZ. Clinical application of sentinel lymph node detection to early stage cervical cancer. Ai Zheng. 2006;25:224-228.

17. Asa S, Sager S, Demirayak G, et al. Comparison of SPECT/CT, gamma probe and methylene blue-dye for sentinel lymph node detection in gynecologic tumours [abstract]. Eur J Nucl Med Mol Imaging. 2013;40(suppl):S393.

18. Buda A, Arosio M, Elisei F, et al. The role of SPECT/CT for sentinel lymph node detection in uterine cancer: analysis of 20 cases and literature review [abstract]. Int J Gynecol Cancer. 2011;21(suppl):S1108.

19. Chernov VVI, Sinilkin IG, Kolomiets LA, Chernyshova AL, Titskaya AA, Zelchan RV. Sentinel lymph node detection in surgical treatment of cervical cancer [abstract]. Eur J Nucl Med Mol Imaging. 2012;39(suppl):S578.

20. Hernandez-Heredia CM, Paredes-Barranco P, Vidal-Sicart S, et al. Contribution of SPECT/CT in the sentinel node detection in early cervical cancer [abstract]. Eur J Nucl Med Mol Imaging. 2013;40(suppl):S217.

21. Hertel H, Makowski L, Tjahjadi M, et al. SPECT/CT improved intraoperative detection of sentinel lymph nodes in vaginal and cervical cancer [abstract]. Arch Gynecol Obstet. 2010;282(suppl):S246-S247.

22. Miyata H, Tsuji N, Hanada T, et al. Sentinel nodes biopsy can accurately predict lymph nodes metastases in early stage cervical cancer, compared with MRI and PET-CT [abstract]. Int J Gynecol Cancer. 2012;22(suppl):E965.

23. Schmidt E, Bozsa S, Gocze $P$, et al. The significance of additional SPECT/CT imaging in sentinel lymph node scintigraphy of patients with cervical and endometrial cancer [abstract]. Eur J Nucl Med Mol Imaging. 2012;39(suppl):S464.

24. Guedec-Ghelfi R, Papathanassiou D, Bruna-Muraille C, et al. Impact of the SPECT/CT in the sentinel lymph node detection: apropos of 32 cases. Med Nucl (Paris). 2011;35:208-218.

25. Kraft O, Havel M. Sentinel lymph nodes and planar scintigraphy and SPECT/CT in various types of tumours: estimation of some factors influencing detection success. Nucl Med Rev Cent East Eur. 2013;16:17-25.

26. Pandit-Taskar N, Gemignani ML, Lyall A, Larson SM, Barakat RR, AbuRustum NR. Single photon emission computed tomography SPECT-CT improves sentinel node detection and localization in cervical and uterine malignancy. Gynecol Oncol. 2010;117:59-64.

27. Buda A, Elisei F, Arosio M, et al. Integration of hybrid single-photon emission computed tomography/computed tomography in the preoperative assessment of sentinel node in patients with cervical and endometrial cancer: our experience and literature review. Int J Gynecol Cancer. 2012;22:830-835.

28. Belhocine TZ, Prefontaine M, Lanvin D, et al. Added-value of SPECT/CT to lymphatic mapping and sentinel lymphadenectomy in gynaecological cancers. Am J Nucl Med Mol Imaging. 2013;3:182-193.

29. Frumovitz M, Ramirez PT, Levenback CF. Lymphatic mapping and sentinel lymph node detection in women with cervical cancer. Gynecol Oncol. 2008; 110(suppl 2):S17-S20.

30. Eiriksson LR, Covens A. Sentinel lymph node mapping in cervical cancer: the future? BJOG. 2012;119:129-133.

31. El-Ghobashy AE, Saidi SA. Sentinel lymph node sampling in gynaecological cancers: techniques and clinical applications. Eur J Surg Oncol. 2009;35:675-685.

32. Roy M, Bouchard-Fortier G, Popa I, et al. Value of sentinel node mapping in cancer of the cervix. Gynecol Oncol. 2011;122:269-274.

33. Cordero García JM, López de la Manzanara-Cano CA, García-Vicente AM, et al. Study of the sentinel node in endometrial cancer at early stages: preliminary results. Rev Esp Med Nucl Imagen Mol. 2012;31:243-248.

34. Gortzak-Uzan L, Jimenez W, Nofech-Mozes S, et al. Sentinel lymph node biopsy vs. pelvic lymphadenectomy in early stage cervical cancer: is it time to change the gold standard? Gynecol Oncol. 2010;116:28-32.

35. Hauspy J, Beiner M, Harley I, Ehrlich L, Rasty G, Covens A. Sentinel lymph nodes in early stage cervical cancer. Gynecol Oncol. 2007;105:285-290.

36. Comparison of pelvic lymphadenectomy versus isolated sentinel lymph node biopsy procedure for early stages of cervical cancers: a randomized multicenter study with evaluation of medico-economic impacts. ClinicalTrials.gov website. http://clinicaltrials.gov/ct2/show/NCT01639820. Updated August 21, 2014. Accessed March 19, 2015.

37. Querleu D, Leblanc E, Cartron G, Narducci F, Ferron G, Martel P. Audit of preoperative and early complications of laparoscopic lymph node dissection in 1000 gynecologic cancer patients. Am J Obstet Gynecol. 2006;195:1287-1292.

38. Achouri A, Huchon C, Bats AS, Bensaid C, Nos C, Lécuru F. Complications of lymphadenectomy for gynecologic cancer. Eur J Surg Oncol. 2013;39:81-86.

39. Hasegawa BH, Wong KH, Iwata K, et al. Dual-modality imaging of cancer with SPECT/CT. Technol Cancer Res Treat. 2002;1:449-458.

40. Buck AK, Nekolla S, Ziegler S, et al. SPECT/CT. J Nucl Med. 2008;49:1305-1319. 